\title{
SOME HERMITE-JENSEN-MERCER LIKE INEQUALITIES FOR CONVEX FUNCTIONS THROUGH A CERTAIN GENERALIZED FRACTIONAL INTEGRALS AND RELATED RESULTS
}

\author{
SAAD IHSAN BUTT, AHMET OCAK AKDEMIR, JAMSHED NASIR, \\ AND FAHD JARAD
}

Received 10 May, 2020

\begin{abstract}
In this article, in light of Jensen-Mercer inequality for functions whose derivatives in the absolute values are convex, some new Hermite-Jensen-Mercer inequalities have been obtained with the help of generalized types of fractional integral operators generated recently by specified local derivatives.
\end{abstract}

2010 Mathematics Subject Classification: 26D15; 26D10; 90C23

Keywords: convex functions, Hermite-Hadamard inequality, Jensen inequality, Jensen-Mercer inequality, fractional integrals

\section{INTRODUCTION}

Convex functions, a function class that has a very useful structure in terms of both definition and features, are a unique and important part of the theory of inequality. This function class has reinforced its importance with its use in inequality theory studies and has been used in various application areas by sourcing new types of inequality. Jensen inequality, which is one of the cornerstones of the theory of inequality, has been the focus of many researchers and many articles on different versions of this famous inequality have been brought into the literature. Jensen-Mercer inequality, a new variant of Jensen inequality introduced for convex functions, is a well known inequality proved for convex functions in theory. A famous inequality proved by using convex functions is called Jensen's inequality and is given as follows. Assume that $0<x_{1} \leq x_{2} \leq \ldots \leq x_{n}$ and $\mu=\left(\mu_{1}, \mu_{2}, \ldots, \mu_{n}\right)$ are non-negative weights such that $\sum_{k=1}^{n} \mu_{k}=1$. The celebrated Jensen inequality (see [16]), in the literature states that $\tau$ is convex functions on the interval $[\theta, \vartheta]$, then

$$
\tau\left(\sum_{k=1}^{n} \mu_{k} x_{k}\right) \leq\left(\sum_{k=1}^{n} \mu_{k} \tau\left(x_{k}\right)\right)
$$


$\forall x_{k} \in[\theta, \vartheta]$ and all $\mu_{k} \in[0,1], \quad(k=1,2, \ldots, n)$.

Now we will remind another important inequality that is obtained by using convex functions. Hermite-Hadamard inequality, which gives the upper and lower limits of the average value of a convex function depending on the averages of real numbers, has been the focus of researchers for nearly a hundred years with its applications in inequality theory, numerical analysis and applied mathematics. Numerous generalizations, expansions, new variants and improvements have been achieved regarding this inequality.

The following statement;

$$
f\left(\frac{a+b}{2}\right) \leq \frac{1}{b-a} \int_{a}^{b} f(x) d x \leq \frac{f(a)+f(b)}{2}
$$

holds and known as Hermite-Hadamard inequality. Both inequalities hold in the reversed direction if $f$ is concave.

There have been many studies on the Jensen inequality, and one of the most notable studies is the Jensen-Mercer inequality, a new version of the Jensen inequality given by Mercer in [17]. Later, inspired by this impressive work, Matkovic et al. JensenMercer generalized the inequality to operators and enriched them with applications (see [16]). Over the years, the Jensen-Mercer inequality has attracted the attention of many researchers, and many studies have been conducted in different ways such as taking this inequality to a higher dimension, obtaining it for superquadratic functions, reverse Jensen-Mercer inequality and finding various generalizations (see the papers $[1-5,9,10,13,19,20,22])$.

Theorem 1 (see [16]). If $\tau$ is a convex function on $[\theta, \vartheta]$, then

$$
\tau\left(\theta+\vartheta-\sum_{k=1}^{n} \mu_{k} x_{k}\right) \leq \tau(\theta)+\tau(\vartheta)-\sum_{k=1}^{n} \mu_{k} \tau\left(x_{k}\right),
$$

$\forall x_{k} \in[\theta, \vartheta]$ and all $\mu_{k} \in[0,1],(k=1,2, \ldots, n)$.

We will proceed with some concepts that will be useful for our findings. Consider the definitions of the Euler Gamma function $\Gamma($.$) and Beta function B(.$, . .), respectively:

$$
\begin{aligned}
& \Gamma(\theta)=\int_{0}^{\infty} e^{-\lambda} \lambda^{\theta-1} d \lambda \\
& B(r, s)=\int_{0}^{1} \lambda^{r-1}(1-\lambda)^{s-1} d \lambda .
\end{aligned}
$$

The solution of some differential equations where the definition of classical derivatives was insufficient remained a problem that mathematicians wondered for years. This curiosity has also pushed mathematicians into a new quest for the real-world applications where classical analysis is desperate. As a result of this search, fractional derivative and integral operators have been identified and studies in fractional analysis 
have gained speed. Today, many different fractional derivative and integral operator definitions and properties are given. The functionality of these operators was revealed by making various comparisons by examining the singularity, locality and some other features of each operator. Some of these operators came to the forefront due to generalizing a few others. Now, we are in a position to recall Riemann-Liouville fractional (RLF) integrals and certain generalized fractional integral operators that generalize Riemann-Liouville, Hadamard and generalized integral operators in a single form.

Definition 1 ([15]). Let $\tau \in[\theta, \vartheta]$. The Riemann-Liouville integrals $J_{\theta+}^{\alpha} \tau$ and $J_{\vartheta-}^{\alpha} \tau$ of order $\alpha>0$ with $\theta, \vartheta \geq 0$ are defined by

$$
\left(J_{\theta+}^{\alpha}\right) \tau(y)=\frac{1}{\Gamma(\alpha)} \int_{\theta}^{y}(y-\lambda)^{\alpha-1} \tau(\lambda) d \lambda ; \quad y>\theta
$$

and

$$
\left(J_{\vartheta-}^{\alpha}\right) \tau(y)=\frac{1}{\Gamma(\alpha)} \int_{y}^{\vartheta}(\lambda-y)^{\alpha-1} \tau(\lambda) d \lambda ; \quad y<\vartheta,
$$

respectively, where $\Gamma($.$) is the Euler Gamma function and \left(J_{\theta+}^{0}\right) \tau(y)=\left(J_{\vartheta-}^{0}\right) \tau(y)=$ $\tau(y)$.

In [12], Jarad et al. have defined new conformable fractional integral operator as follows:

$$
{ }_{\theta}^{\beta} J^{\alpha} \tau(y)=\frac{1}{\Gamma(\beta)} \int_{\theta}^{y}\left(\frac{(y-\theta)^{\alpha}-(\lambda-\theta)^{\alpha}}{\alpha}\right)^{\beta-1} \frac{\tau(\lambda)}{(\lambda-\theta)^{1-\alpha}} d \lambda,
$$

and

$$
\beta J_{\vartheta}^{\alpha} \tau(y)=\frac{1}{\Gamma(\beta)} \int_{y}^{\vartheta}\left(\frac{(\vartheta-y)^{\alpha}-(\vartheta-\lambda)^{\alpha}}{\alpha}\right)^{\beta-1} \frac{\tau(\lambda)}{(\vartheta-\lambda)^{1-\alpha}} d \lambda .
$$

New conformable fractional integral operator has some special cases and connections according to special values of parameters as follows:

Remark 1. i) If we set $\theta=0$ and $\alpha=1$ in (1.5), then the integral operator that is defined with the equation (1.5) reduces to the Riemann-Liouville integral operator that is given in (1.3).

ii) If we choose $\theta=0$ and $\alpha \rightarrow 0$, the equation (1.5) coincides with the Hadamard fractional integral that is defined in [14]. Also, if we select $\theta=0$, the new fractional integral operator reduces to Katugampola fractional integral operator.

iii) Similar connecitons can be established for (1.6). The operator that is defined in (1.6) reduces to the Riemann-Liouville integral operator that is given in (1.4), if we set $\vartheta=0$ and $\alpha=1$. It also coincide with the Hadamard fractional integral that is given in [14] when we set $\vartheta=0$ and $\alpha \rightarrow 0$ in (1.6).

For more results in inequality theory and various operators in fractional analysis and detailed information on these operators, we recommend readers ([6-8,11, 12, 14, $15,18,23-25])$. 
The main motivation in this study is to obtain new Jensen-Mercer-like HermiteHadamard type inequalities by using fractional integrals defined in (1.5) and (1.6) based on Jensen-Mercer inequality for functions whose derivatives are convex. Then, with the help of Hölder inequality and useful variants, inequalities similar to JensenMercer inequality were obtained. Many special cases have been demonstrated to verify inequalities in the literature.

\section{HeRmite-JENSEN-MERCER LIKE INEQUALITIES FOR NEW CONFORMABLE FRACTIONAL INTEGRALS}

With the help of the operator known as the fractional integral introduced as a new generalization in fractional analysis, an Hermite-Jensen-Mercer type inequality is given as follows:

Theorem 2. Suppose that $\tau:[\theta, \vartheta] \rightarrow R$ is a convex mapping. Then the following inequality for fractional integrals holds:

$$
\begin{aligned}
& \tau\left(\theta+\vartheta-\frac{x+y}{2}\right) \leq \frac{2^{\alpha \beta-1} \alpha^{\beta} \Gamma(\beta+1)}{(y-x)^{\alpha \beta}} \\
& \times\left\{\begin{array}{l}
\beta \\
\left(\theta+\vartheta-\frac{x+y}{2}\right)
\end{array} J^{\alpha} \tau(\theta+\vartheta-x)+{ }^{\beta} J_{\left(\theta+\vartheta-\frac{x+y}{2}\right)}^{\alpha} \tau(\theta+\vartheta-y)\right\} \\
& \leq \tau(\theta)+\tau(\vartheta)-\left(\frac{\tau(x)+\tau(y)}{2}\right),
\end{aligned}
$$

where $\forall x, y \in[\theta, \vartheta], \alpha, \beta>0$ and $\Gamma($.$) is the Euler Gamma function.$

Proof. Let's start proving the first part of inequality with the fact that $\tau$ is convex, then we can write

$$
\begin{aligned}
& \tau\left(\theta+\vartheta-\frac{x_{1}+y_{1}}{2}\right)=\tau\left(\frac{\theta+\vartheta-x_{1}+\theta+\vartheta-y_{1}}{2}\right) \\
& \leq \frac{\tau\left(\theta+\vartheta-x_{1}\right)+\tau\left(\theta+\vartheta-y_{1}\right)}{2},
\end{aligned}
$$

$\forall x_{1}, y_{1} \in[\theta, \vartheta]$. Hence if we set $x_{1}=\frac{\lambda}{2} x+\frac{2-\lambda}{2} y$ and $y_{1}=\frac{2-\lambda}{2} x+\frac{\lambda}{2} y$, for $x, y \in[\theta, \vartheta]$ and $\lambda \in[0,1]$, we get

$$
\begin{aligned}
2 \tau\left(\theta+\vartheta-\frac{x+y}{2}\right) & \leq \tau\left(\theta+\vartheta-\left(\frac{\lambda}{2} x+\frac{2-\lambda}{2} y\right)\right) \\
& +\tau\left(\theta+\vartheta-\left(\frac{2-\lambda}{2} x+\frac{\lambda}{2} y\right)\right) .
\end{aligned}
$$


Now, to combine the above inequality with the operator, we multiply both sides of (2.2) by $\left(\frac{1-(1-\lambda)^{\alpha}}{\alpha}\right)^{\beta-1}(1-\lambda)^{\alpha-1}$ and then integrating the resulting inequality with respect to $\lambda$ over $[0,1]$, we have

$$
\begin{aligned}
& 2 \tau\left(\theta+\vartheta-\frac{x+y}{2}\right) \int_{0}^{1}\left(\frac{1-(1-\lambda)^{\alpha}}{\alpha}\right)^{\beta-1}(1-\lambda)^{\alpha-1} d \lambda \\
& \leq \int_{0}^{1}\left(\frac{1-(1-\lambda)^{\alpha}}{\alpha}\right)^{\beta-1}(1-\lambda)^{\alpha-1} \\
& \times\left(\tau\left(\theta+\vartheta-\left(\frac{\lambda}{2} x+\frac{2-\lambda}{2} y\right)\right)+\tau\left(\theta+\vartheta-\left(\frac{2-\lambda}{2} x+\frac{\lambda}{2} y\right)\right)\right) d \lambda \\
& =\left(\frac{2}{y-x}\right)^{\alpha \beta}\left\{\Gamma(\beta)^{\beta} J_{\left(\theta+\vartheta-\frac{x+y}{2}\right.}^{\alpha} \tau(\theta+\vartheta-y)+\Gamma(\beta)_{\left(\theta+\vartheta-\frac{x+y}{2}\right.}^{\beta} J^{\alpha} \tau(\theta+\vartheta-x)\right\} .
\end{aligned}
$$

It is obvious that

$$
\int_{0}^{1}\left(\frac{1-(1-\lambda)^{\alpha}}{\alpha}\right)^{\beta-1}(1-\lambda)^{\alpha-1} d \lambda=\frac{1}{\beta \alpha^{\beta}}
$$

As a consequence,

$$
\begin{aligned}
& 2 \tau\left(\theta+\vartheta-\frac{x+y}{2}\right) \frac{1}{\beta \alpha^{\beta}} \\
& \leq\left(\frac{2}{y-x}\right)^{\alpha \beta}\left\{\Gamma(\beta){ }^{\beta} J_{\left(\theta+\vartheta-\frac{x+y}{2}\right.}^{\alpha} \tau(\theta+\vartheta-y)+\Gamma(\beta)_{\left(\theta+\vartheta-\frac{x+y}{2}\right)}^{\beta} J^{\alpha} \tau(\theta+\vartheta-x)\right\} .
\end{aligned}
$$

Thus, this completes the proof of the first inequality of (2).

Again, to prove the second part, we remember that $\tau$ is convex mapping, then for $\lambda \in[0,1]$, we can write

$$
\tau\left(\theta+\vartheta-\left(\frac{\lambda}{2} x+\frac{2-\lambda}{2} y\right)\right) \leq \tau(\theta)+\tau(\vartheta)-\left(\frac{\lambda}{2} \tau(x)+\frac{2-\lambda}{2} \tau(y)\right),
$$

and

$$
\tau\left(\theta+\vartheta-\left(\frac{2-\lambda}{2} x+\frac{\lambda}{2} y\right)\right) \leq \tau(\theta)+\tau(\vartheta)-\left(\frac{2-\lambda}{2} \tau(x)+\frac{\lambda}{2} \tau(y)\right) .
$$

By adding the inequalities of (2.4) and (2.5), we have

$$
\begin{aligned}
& \tau\left(\theta+\vartheta-\left(\frac{\lambda}{2} x+\frac{2-\lambda}{2} y\right)\right)+\tau\left(\theta+\vartheta-\left(\frac{2-\lambda}{2} x+\frac{\lambda}{2} y\right)\right) \\
& \leq 2(\tau(\theta)+\tau(\vartheta))-(\tau(x)+\tau(y)) .
\end{aligned}
$$


Now multiplying (2.6) by $\left(\frac{1-(1-\lambda)^{\alpha}}{\alpha}\right)^{\beta-1}(1-\lambda)^{\alpha-1}$ and then integrating the resulting inequality with respect to $\lambda$ over $[0,1]$, we obtain

$$
\begin{aligned}
& \int_{0}^{1}\left(\frac{1-(1-\lambda)^{\alpha}}{\alpha}\right)^{\beta-1}(1-\lambda)^{\alpha-1} \\
& \times\left\{\tau\left(\theta+\vartheta-\left(\frac{\lambda}{2} x+\frac{2-\lambda}{2} y\right)\right)+\tau\left(\theta+\vartheta-\left(\frac{2-\lambda}{2} x+\frac{\lambda}{2} y\right)\right)\right\} d \lambda \\
& \leq\{2(\tau(\theta)+\tau(\vartheta))-(\tau(x)+\tau(y))\} \int_{0}^{1}\left(\frac{1-(1-\lambda)^{\alpha}}{\alpha}\right)^{\beta-1}(1-\lambda)^{\alpha-1} d \lambda .
\end{aligned}
$$

Namely,

$$
\begin{aligned}
& \left(\frac{2}{y-x}\right)^{\alpha \beta}\left\{\Gamma(\beta)^{\beta} J_{\left(\theta+\vartheta-\frac{x+y}{2}\right)}^{\alpha} \tau(\theta+\vartheta-y)+\Gamma(\beta){ }_{\left(\theta+\vartheta-\frac{x+y}{2}\right)}^{\beta} J^{\alpha} \tau(\theta+\vartheta-x)\right\} \\
& \leq \frac{1}{\beta \alpha^{\beta}}\{2(\tau(\theta)+\tau(\vartheta))-(\tau(x)+\tau(y))\} .
\end{aligned}
$$

This completes the proof.

Remark 2. (i) If we take $x=\theta$ and $y=\vartheta$ in Theorem 2 , then we get (Theorem $2.1,[11])$.

(ii) If we take $\alpha=1, x=\theta$ and $y=\vartheta$ in Theorem 2, then we get (Theorem 2, [24]).

Theorem 3. Suppose that $\tau:[\theta, \vartheta] \rightarrow R$ be a convex mapping. Then the following inequality for fractional integrals holds:

$$
\begin{aligned}
& \tau\left(\theta+\vartheta-\frac{x+y}{2}\right) \leq \tau(\theta)+\tau(\vartheta)-\frac{\alpha^{\beta} \Gamma(\beta+1)}{2(y-x)^{\alpha \beta}}\left\{\beta J_{x}^{\alpha} \tau(y)+{ }_{y}^{\beta} J^{\alpha} \tau(x)\right\} \\
& \leq \tau(\theta)+\tau(\vartheta)-\tau\left(\frac{x+y}{2}\right)
\end{aligned}
$$

and

$$
\begin{aligned}
& \tau\left(\theta+\vartheta-\frac{x+y}{2}\right) \\
& \leq \frac{\alpha^{\beta} \Gamma(\beta+1)}{2(y-x)^{\alpha \beta}}\left\{{ }^{\beta} J_{\theta+\vartheta-x}^{\alpha} \tau(\theta+\vartheta-y)+{ }_{\theta+\vartheta-y}^{\beta} J^{\alpha} \tau(\theta+\vartheta-x)\right\} \\
& \leq \frac{\tau(\theta+\vartheta-x)+\tau(\theta+\vartheta-y)}{2} \leq \tau(\theta)+\tau(\vartheta)-\frac{\tau(x)+\tau(y)}{2}
\end{aligned}
$$

where $\forall x, y \in[\theta, \vartheta], \alpha, \beta>0$ and $\Gamma($.$) is the Euler Gamma function.$ 
Proof. By making use of the Jensen-Mercer inequality, we get

$$
\tau\left(\theta+\vartheta-\frac{x_{1}+y_{1}}{2}\right) \leq \tau(\theta)+\tau(\vartheta)-\frac{\tau\left(x_{1}\right)+\tau\left(y_{1}\right)}{2},
$$

$\forall x_{1}, y_{1} \in[\theta, \vartheta]$. By changing of variables $x_{1}=\lambda x+(1-\lambda) y$ and $y_{1}=(1-\lambda) x+\lambda y$, for $x, y \in[\theta, \vartheta]$ and $\lambda \in[0,1]$ in (2.9), we can write

$$
\tau\left(\theta+\vartheta-\frac{x+y}{2}\right) \leq \tau(\theta)+\tau(\vartheta)-\frac{\tau(\lambda x+(1-\lambda) y)+\tau((1-\lambda) x+\lambda y)}{2} .
$$

Now by multiplying (2.10) by $\left(\frac{1-(1-\lambda)^{\alpha}}{\alpha}\right)^{\beta-1}(1-\lambda)^{\alpha-1}$ and then integrating the resulting inequality with respect to $\lambda$ over $[0,1]$, we success to reach the statement of new conformable fractional operator;

$$
\begin{aligned}
& \tau\left(\theta+\vartheta-\frac{x+y}{2}\right) \int_{0}^{1}\left(\frac{1-(1-\lambda)^{\alpha}}{\alpha}\right)^{\beta}(1-\lambda)^{\alpha-1} d \lambda \\
& \leq \int_{0}^{1}\left(\frac{1-(1-\lambda)^{\alpha}}{\alpha}\right)^{\beta}(1-\lambda)^{\alpha-1} \\
& \times\left\{\tau(\theta)+\tau(\vartheta)-\frac{\tau(\lambda x+(1-\lambda) y)+\tau((1-\lambda) x+\lambda y)}{2}\right\} d \lambda .
\end{aligned}
$$

By making some simplyfing processes, we obtain

$$
\tau\left(\theta+\vartheta-\frac{x+y}{2}\right) \leq \tau(\theta)+\tau(\vartheta)-\frac{\alpha^{\beta} \Gamma(\beta+1)}{2(y-x)^{\alpha \beta}}\left\{\beta J_{x}^{\alpha} \tau(y)+{ }_{y}^{\beta} J^{\alpha} \tau(x)\right\} .
$$

This completes the proof of the first inequality of (2.7).

Now, for the proof of second inequality of (2.7), we first note that if $\tau$ is convex function, then for $\lambda \in[0,1]$, we obtain

$$
\begin{aligned}
\tau\left(\frac{x+y}{2}\right) & =\tau\left(\frac{\lambda x+(1-\lambda) y+(1-\lambda) x+\lambda y}{2}\right) \\
\leq & \frac{\tau(\lambda x+(1-\lambda) y)+\tau((1-\lambda) x+\lambda y)}{2}
\end{aligned}
$$


By multiplying (2.12) by $\left(\frac{1-(1-\lambda)^{\alpha}}{\alpha}\right)^{\beta-1}(1-\lambda)^{\alpha-1}$ and then integrating the resulting inequality with respect to $\lambda$ over $[0,1]$, we have

$$
\begin{aligned}
& \tau\left(\frac{x+y}{2}\right) \int_{0}^{1}\left(\frac{1-(1-\lambda)^{\alpha}}{\alpha}\right)^{\beta}(1-\lambda)^{\alpha-1} d \lambda \\
& \leq \int_{0}^{1}\left(\frac{1-(1-\lambda)^{\alpha}}{\alpha}\right)^{\beta}(1-\lambda)^{\alpha-1}\left\{\frac{\tau(\lambda x+(1-\lambda) y)+\tau((1-\lambda) x+\lambda y)}{2}\right\} d \lambda,
\end{aligned}
$$

after simplification and change of variables, we get

$$
\begin{aligned}
& \tau\left(\frac{x+y}{2}\right) \leq \frac{\alpha^{\beta} \Gamma(\beta+1)}{2(y-x)^{\alpha \beta}}\left\{\beta J_{x}^{\alpha} \tau(y)+{ }_{y}^{\beta} J^{\alpha} \tau(x)\right\} \\
& -\tau\left(\frac{x+y}{2}\right) \geq-\frac{\alpha^{\beta} \Gamma(\beta+1)}{2(y-x)^{\alpha \beta}}\left\{\beta J_{x}^{\alpha} \tau(y)+{ }_{y}^{\beta} J^{\alpha} \tau(x)\right\}
\end{aligned}
$$

adding $\tau(\theta)+\tau(\vartheta)$ to both side of (2.13), we obtain

$$
\begin{aligned}
& \tau(\theta)+\tau(\vartheta)-\tau\left(\frac{x+y}{2}\right) \\
& \geq \tau(\theta)+\tau(\vartheta)-\frac{\alpha^{\beta} \Gamma(\beta+1)}{2(y-x)^{\alpha \beta}}\left\{\beta J_{x}^{\alpha} \tau(y)+{ }_{y}^{\beta} J^{\alpha} \tau(x)\right\} .
\end{aligned}
$$

By combining (2.11) and (2.14), we prove (2.7). To prove (2.8), we will start with the convexity of $\tau$,

$$
\begin{aligned}
\tau\left(\theta+\vartheta-\frac{x_{1}+y_{1}}{2}\right) & =\tau\left(\frac{\theta+\vartheta-x_{1}+\theta+\vartheta-y_{1}}{2}\right) \\
\leq & \frac{\tau\left(\theta+\vartheta-x_{1}\right)+\tau\left(\theta+\vartheta-y_{1}\right)}{2}
\end{aligned}
$$

$\forall x_{1}, y_{1} \in[\theta, \vartheta]$. By change of variables such that $x_{1}=\lambda x+(1-\lambda) y$ and $y_{1}=$ $(1-\lambda) x+\lambda y$, for $x, y \in[\theta, \vartheta]$ and $\lambda \in[0,1]$ in $(2.15)$, we get

$$
\begin{aligned}
& \tau\left(\theta+\vartheta-\frac{x+y}{2}\right) \\
& \leq\left\{\frac{\tau(\theta+\vartheta-(\lambda x+(1-\lambda) y))+\tau(\theta+\vartheta-((1-\lambda) x+\lambda y))}{2}\right\} .
\end{aligned}
$$


To combine the above inequality with the operator, we multiply (2.16) by $\left(\frac{1-(1-\lambda)^{\alpha}}{\alpha}\right)^{\beta-1}(1-\lambda)^{\alpha-1}$ and then integrating the resulting inequality with respect to $\lambda$ over $[0,1]$, we have

$$
\begin{aligned}
& \tau\left(\theta+\vartheta-\frac{x+y}{2}\right) \int_{0}^{1}\left(\frac{1-(1-\lambda)^{\alpha}}{\alpha}\right)^{\beta}(1-\lambda)^{\alpha-1} d \lambda \\
& \leq \int_{0}^{1}\left(\frac{1-(1-\lambda)^{\alpha}}{\alpha}\right)^{\beta}(1-\lambda)^{\alpha-1} \\
& \times\left\{\frac{\tau(\theta+\vartheta-(\lambda x+(1-\lambda) y))+\tau(\theta+\vartheta-((1-\lambda) x+\lambda y))}{2}\right\} d \lambda,
\end{aligned}
$$

By making use of the necessary operations, we get

$$
\begin{aligned}
& \tau\left(\theta+\vartheta-\frac{x+y}{2}\right) \\
& \leq \frac{\alpha^{\beta} \Gamma(\beta+1)}{2(y-x)^{\alpha \beta}}\left\{\beta J_{\theta+\vartheta-x}^{\alpha} \tau(\theta+\vartheta-y)+{ }_{\theta+\vartheta-y}^{\beta} J^{\alpha} \tau(\theta+\vartheta-x)\right\}
\end{aligned}
$$

On the other hand, using the convexity of $\tau$, we can write

$$
\tau(\lambda(\theta+\vartheta-x)+(1-\lambda)(\theta+\vartheta-y)) \leq \lambda \tau(\theta+\vartheta-x)+(1-\lambda) \tau(\theta+\vartheta-y)
$$

and

$$
\tau((1-\lambda)(\theta+\vartheta-x)+\lambda(\theta+\vartheta-y)) \leq(1-\lambda) \tau(\theta+\vartheta-x)+\lambda \tau(\theta+\vartheta-y)
$$

By adding above two inequalities and using the celebrated Jensen-Mercer inequality, we have

$$
\begin{aligned}
& \tau(\lambda(\theta+\vartheta-x)+(1-\lambda)(\theta+\vartheta-y))+\tau((1-\lambda)(\theta+\vartheta-x)+\lambda(\theta+\vartheta-y)) \\
& \leq \tau(\theta+\vartheta-x)+\tau(\theta+\vartheta-y) \leq 2(\tau(\theta)+\tau(\vartheta))-(\tau(x)+\tau(y)) .
\end{aligned}
$$

By multiplying (2.18) by $\left(\frac{1-(1-\lambda)^{\alpha}}{\alpha}\right)^{\beta-1}(1-\lambda)^{\alpha-1}$ and then integrating the resulting 
inequality with respect to $\lambda$ over $[0,1]$, we have

$$
\begin{aligned}
& \int_{0}^{1}\left(\frac{1-(1-\lambda)^{\alpha}}{\alpha}\right)^{\beta}(1-\lambda)^{\alpha-1}\{\tau(\lambda(\theta+\vartheta-x)+(1-\lambda)(\theta+\vartheta-y)) \\
& +\tau((1-\lambda)(\theta+\vartheta-x)+\lambda(\theta+\vartheta-y))\} d \lambda \\
& \leq 2(\tau(\theta)+\tau(\vartheta))-(\tau(x)+\tau(y)) \int_{0}^{1}\left(\frac{1-(1-\lambda)^{\alpha}}{\alpha}\right)^{\beta}(1-\lambda)^{\alpha-1} d \lambda,
\end{aligned}
$$

Again some basic operations, we get

$$
\begin{aligned}
& \frac{\alpha^{\beta} \Gamma(\beta+1)}{2(y-x)^{\alpha \beta}}\left\{\beta J_{\theta+\vartheta-x}^{\alpha} \tau(\theta+\vartheta-y)+{ }_{\theta+\vartheta-y}^{\beta} J^{\alpha} \tau(\theta+\vartheta-x)\right\} \\
& \leq(\tau(\theta)+\tau(\vartheta))-\left(\frac{\tau(x)+\tau(y)}{2}\right),
\end{aligned}
$$

Finally, by combining (2.17) and (2.19), we get (2.8). Which completes the proof.

Remark 3. Under the assumptions of Theorem 3 with $\alpha=\beta=1$, one has

$$
\text { (i) } \begin{aligned}
& \tau\left(\theta+\vartheta-\frac{x+y}{2}\right) \leq \tau(\theta)+\tau(\vartheta)-\int_{0}^{1} \tau(\lambda x+(1-\lambda) y) d \lambda \\
& \leq \tau(\theta)+\tau(\vartheta)-\tau\left(\frac{x+y}{2}\right)
\end{aligned}
$$

and

$$
\text { (ii) } \begin{aligned}
& \tau\left(\theta+\vartheta-\frac{x+y}{2}\right) \leq \frac{1}{y-x} \int_{x}^{y} \tau(\theta+\vartheta-\lambda) d \lambda \\
& \leq \tau(\theta)+\tau(\vartheta)-\frac{\tau(x)+\tau(y)}{2} .
\end{aligned}
$$

The Remark 3 is proved in (Theorem 2.1, [13]).

Lemma 1. Assume that $\tau:[\theta, \vartheta] \rightarrow R$ is differentiable mapping on $(\theta, \vartheta)$ with $\theta<\vartheta$. If $\tau^{\prime} \in L[\theta, \vartheta]$, then the following equation holds for fractional integrals:

$$
\begin{aligned}
& \frac{2^{\alpha \beta-1} \alpha^{\beta} \Gamma(\beta+1)}{(y-x)^{\alpha \beta}} \\
& \times\left\{\begin{array}{l}
\beta \\
\left(\theta+\vartheta-\frac{x+y}{2}\right)
\end{array} J^{\alpha} \tau(\theta+\vartheta-x)+{ }^{\beta} J_{\left(\theta+\vartheta-\frac{x+y}{2}\right)}^{\alpha} \tau(\theta+\vartheta-y)\right\} \\
& -\tau\left(\theta+\vartheta-\frac{x+y}{2}\right)
\end{aligned}
$$




$$
\begin{aligned}
& =\frac{(y-x) \alpha^{\beta}}{4} \int_{0}^{1}\left(\frac{1-(1-\lambda)^{\alpha}}{\alpha}\right)^{\beta} \\
& \times\left\{\tau^{\prime}\left(\theta+\vartheta-\left(\frac{2-\lambda}{2} x+\frac{\lambda}{2} y\right)\right)-\tau^{\prime}\left(\theta+\vartheta-\left(\frac{\lambda}{2} x+\frac{2-\lambda}{2} y\right)\right)\right\} d \lambda
\end{aligned}
$$

where $\forall x, y \in[\theta, \vartheta], \alpha, \beta>0$ and $\Gamma($.$) is the Euler Gamma function.$

Proof. It suffices to note that,

$$
I=\frac{y-x}{4} \alpha^{\beta}\left\{I_{1}-I_{2}\right\}
$$

where

$$
I_{1}=\int_{0}^{1}\left(\frac{1-(1-\lambda)^{\alpha}}{\alpha}\right)^{\beta} \tau^{\prime}\left(\theta+\vartheta-\left(\frac{2-\lambda}{2} x+\frac{\lambda}{2} y\right)\right) d \lambda
$$

and

$$
I_{2}=\int_{0}^{1}\left(\frac{1-(1-\lambda)^{\alpha}}{\alpha}\right)^{\beta} \tau^{\prime}\left(\theta+\vartheta-\left(\frac{\lambda}{2} x+\frac{2-\lambda}{2} y\right)\right) d \lambda
$$

Integrating by parts, we have

$$
\begin{aligned}
I_{1} & =-\frac{2}{\alpha^{\beta}(y-x)} \tau\left(\theta+\vartheta-\frac{x+y}{2}\right) \\
& +\frac{2 \alpha \beta}{\alpha^{\beta}(y-x)} \int_{0}^{1}\left(1-(1-\lambda)^{\alpha}\right)^{\beta-1}(1-\lambda)^{\alpha-1} \tau\left(\theta+\vartheta-\left(\frac{2-\lambda}{2} x+\frac{\lambda}{2} y\right)\right) d \lambda \\
& =-\frac{2}{\alpha^{\beta}(y-x)} \tau\left(\theta+\vartheta-\frac{x+y}{2}\right) \\
& +\frac{2^{\alpha \beta} \beta}{\alpha^{\beta-1}(y-x)^{\alpha \beta+1}} \int_{\theta+\vartheta-\frac{x+y}{2}}^{\theta+\vartheta-x}\left(\left(\frac{y-x}{2}\right)^{\alpha}-\left(\lambda_{1}-\left(\theta+\vartheta-\frac{x+y}{2}\right)\right)^{\alpha}\right)^{\beta-1} \\
& \times \frac{\tau\left(\lambda_{1}\right)}{\left(\lambda_{1}-\left(\theta+\vartheta-\frac{x+y}{2}\right)\right)^{1-\alpha}} d \lambda \\
& =-\frac{2}{\alpha^{\beta}(y-x)} \tau\left(\theta+\vartheta-\frac{x+y}{2}\right) \\
& +\left(\frac{2}{y-x}\right)^{\alpha \beta+1} \frac{\Gamma(\beta+1)}{\alpha^{\beta-1}}{ }_{\left(\theta+\vartheta-\frac{x+y}{2}\right.}^{\beta} J^{\alpha} \tau(\theta+\vartheta-x) .
\end{aligned}
$$


Similarly,

$$
\begin{aligned}
& I_{2}= \int_{0}^{1}\left(\frac{1-(1-\lambda)^{\alpha}}{\alpha}\right)^{\beta} \tau^{\prime}\left(\theta+\vartheta-\left(\frac{\lambda}{2} x+\frac{2-\lambda}{2} y\right)\right) d \lambda . \\
& I_{2}=\frac{2}{\alpha^{\beta}(y-x)} \tau\left(\theta+\vartheta-\frac{x+y}{2}\right) \\
&-\left(\frac{2}{y-x}\right)^{\alpha \beta+1} \frac{\Gamma(\beta+1)}{\alpha^{\beta-1}} \beta J_{\left(\theta+\vartheta-\frac{x+y}{2}\right)}^{\alpha} \tau(\theta+\vartheta-y) .
\end{aligned}
$$

By connecting the equations (2.23) and (2.24) with (2.21) and get (2.20). Thus the proof is completed.

Remark 4. (i) If we take $x=\theta$ and $y=\vartheta$, then we can get (Lemma 3.1, [11]).

(ii) If we take $\alpha=1$ and $x=\theta, y=\vartheta$ in Lemma 1, it reduces to (Lemma 1.1, [25]).

Lemma 2. Assume that $\tau:[\theta, \vartheta] \rightarrow R$ is differentiable mapping on $(\theta, \vartheta)$ with $\theta<\vartheta$. If $\tau^{\prime} \in L[\theta, \vartheta]$, then the following equation holds for fractional integrals:

$$
\begin{aligned}
& \frac{\tau(\theta+\vartheta-x)+\tau(\theta+\vartheta-y)}{2}-\frac{\alpha^{\beta} \Gamma(\beta+1)}{2(y-x)^{\alpha \beta}} \\
& \times\left\{\begin{array}{l}
\beta \\
(\theta+\vartheta-y)
\end{array} J^{\alpha} \tau(\theta+\vartheta-x)+{ }^{\beta} J_{(\theta+\vartheta-x)}^{\alpha} \tau(\theta+\vartheta-y)\right\} \\
& =\frac{(y-x) \alpha^{\beta}}{2} \int_{0}^{1}\left[\left(\frac{1-(1-\lambda)^{\alpha}}{\alpha}\right)^{\beta}-\left(\frac{1-\lambda^{\alpha}}{\alpha}\right)^{\beta}\right] \\
& \times \tau^{\prime}(\theta+\vartheta-(\lambda x+(1-\lambda) y)) d \lambda,
\end{aligned}
$$

where $\forall x, y \in[\theta, \vartheta], \alpha, \beta>0$ and $\Gamma($.$) is the Euler Gamma function.$

Proof.

$$
\begin{gathered}
\frac{(y-x) \alpha^{\beta}}{2} \int_{0}^{1}\left[\left(\frac{1-(1-\lambda)^{\alpha}}{\alpha}\right)^{\beta}-\left(\frac{1-\lambda^{\alpha}}{\alpha}\right)^{\beta}\right] \\
\times \tau^{\prime}(\theta+\vartheta-(\lambda x+(1-\lambda) y)) d \lambda . \\
I=\frac{(y-x) \alpha^{\beta}}{2}\left\{I_{1}-I_{2}\right\} .
\end{gathered}
$$




$$
\begin{aligned}
& I_{1}=\int_{0}^{1}\left(\frac{1-(1-\lambda)^{\alpha}}{\alpha}\right)^{\beta} \tau^{\prime}(\theta+\vartheta-(\lambda x+(1-\lambda) y)) d \lambda \\
& =\frac{1}{\alpha^{\beta}} \frac{\tau(\theta+\vartheta-x)}{y-x}-\frac{\beta}{y-x} \int_{0}^{1}\left(\frac{1-(1-\lambda)^{\alpha}}{\alpha}\right)^{\beta-1} \\
& \times(1-\lambda)^{\alpha-1} \tau(\theta+\vartheta-(\lambda x+(1-\lambda) y)) d \lambda \\
& =\frac{1}{\alpha^{\beta}} \frac{\tau(\theta+\vartheta-x)}{y-x}-\frac{\Gamma(\beta+1)}{(y-x)^{\alpha \beta+1}}\left\{\beta J_{\theta+\vartheta-x}^{\alpha} \tau(\theta+\vartheta-y)\right\} . \\
& I_{2}=\int_{0}^{1}\left(\frac{1-\lambda^{\alpha}}{\alpha}\right)^{\beta} \tau^{\prime}(\theta+\vartheta-(\lambda x+(1-\lambda) y)) d \lambda \\
& =-\frac{1}{\alpha^{\beta}} \frac{\tau(\theta+\vartheta-y)}{y-x}+\frac{\Gamma(\beta+1)}{(y-x)^{\alpha \beta+1}}\left\{\begin{array}{l}
\beta \\
\theta+\vartheta-y
\end{array} J^{\alpha} \tau(\theta+\vartheta-x)\right\},
\end{aligned}
$$

combining equations (2.26) and (2.27) with (2.25) and get (2.25). Which completes the proof.

Corollary 1. If we take $\alpha=\beta=1$ in Lemma 2, the we have the following equality:

$$
\begin{aligned}
& \frac{\tau(\theta+\vartheta-x)+\tau(\theta+\vartheta-y)}{2}-\frac{1}{y-x} \int_{\theta+\vartheta-y}^{\theta+\vartheta-x} \tau(\lambda) d \lambda \\
& =\frac{y-x}{2} \int_{0}^{1}(2 \lambda-1) \tau^{\prime}(\theta+\vartheta-(\lambda x+(1-\lambda) y)) d \lambda .
\end{aligned}
$$

Remark 5. If we take $x=\theta$ and $y=\vartheta$ in Corollary 1 , then the equality (2.28) reduces to the equality as following

$$
\frac{\tau(\theta)+\tau(\vartheta)}{2}-\frac{1}{\vartheta-\theta} \int_{\theta}^{\vartheta} \tau(\lambda) d \lambda=\frac{\vartheta-\theta}{2} \int_{0}^{1}(2 \lambda-1) \tau^{\prime}((1-\lambda) \theta+\lambda \vartheta) d \lambda,
$$

which is proved in (Lemma 2.1, [6]).

Theorem 4. $\tau:[\theta, \vartheta] \rightarrow R$ is differentiable mapping on $(\theta, \vartheta)$ with $\theta<\vartheta$ and $\tau^{\prime} \in L[\theta, \vartheta]$. If $\left|\tau^{\prime}\right|$ is convex function on $[\theta, \vartheta]$, then the following inequality holds for fractional integrals:

$$
\begin{aligned}
& \mid \frac{2^{\alpha \beta-1} \alpha^{\beta} \Gamma(\beta+1)}{(y-x)^{\alpha \beta}}\left\{\begin{array}{l}
\beta \\
\left(\theta+\vartheta-\frac{x+y}{2}\right)
\end{array} J^{\alpha} \tau(\theta+\vartheta-x)+\beta J_{\left(\theta+\vartheta-\frac{x+y}{2}\right)}^{\alpha} \tau(\theta+\vartheta-y)\right\} \\
& -\tau\left(\theta+\vartheta-\frac{x+y}{2}\right) \mid
\end{aligned}
$$




$$
\begin{aligned}
& \leq \frac{y-x}{4} \alpha^{\beta}\left[\left(\left|\tau^{\prime}(\theta)\right|+\left|\tau^{\prime}(\vartheta)\right|\right)\left(\frac{1}{\alpha^{\beta+1}} B\left(\beta+1, \frac{1}{\alpha}\right)\right)\right. \\
& -\left\{\left|\tau^{\prime}(x)\right|\left(\frac{1}{2 \alpha^{\beta+1}}\left(B\left(\beta+1, \frac{2}{\alpha}\right)+B\left(\beta+1, \frac{1}{\alpha}\right)\right)\right)\right\} \\
& +\left\{\left|\tau^{\prime}(y)\right|\left(\frac{1}{2 \alpha^{\beta+1}}\left(B\left(\beta+1, \frac{2}{\alpha}\right)-B\left(\beta+1, \frac{1}{\alpha}\right)\right)\right)\right\} \\
& +\left(\left|\tau^{\prime}(\theta)\right|+\left|\tau^{\prime}(\vartheta)\right|\right)\left(\frac{1}{\alpha^{\beta+1}} B\left(\beta+1, \frac{1}{\alpha}\right)\right) \\
& -\left\{\left|\tau^{\prime}(x)\right|\left(\frac{1}{2 \alpha^{\beta+1}}\left(B\left(\beta+1, \frac{1}{\alpha}\right)-B\left(\beta+1, \frac{2}{\alpha}\right)\right)\right)\right\} \\
& \left.+\left\{\left|\tau^{\prime}(y)\right|\left(\frac{1}{2 \alpha^{\beta+1}}\left(B\left(\beta+1, \frac{2}{\alpha}\right)+B\left(\beta+1, \frac{1}{\alpha}\right)\right)\right)\right\}\right]
\end{aligned}
$$

where $\forall x, y \in[\theta, \vartheta], \alpha, \beta>0, \lambda \in[0,1]$ and $\Gamma(),. B(.,$.$) are the Euler Gamma$ and Beta function.

Proof. By using Lemma 1 with Jensen-Mercer inequality and the convexity of $\left|\tau^{\prime}\right|$, we can write

$$
\begin{aligned}
& \quad \mid \frac{2^{\alpha \beta-1} \alpha^{\beta} \Gamma(\beta+1)}{(y-x)^{\alpha \beta}\left\{\begin{array}{l}
\beta \\
\left(\theta+\vartheta-\frac{x+y}{2}\right.
\end{array} J^{\alpha} \tau(\theta+\vartheta-x)+{ }^{\beta} J_{\left(\theta+\vartheta-\frac{x+y}{2}\right.}^{\alpha} \tau(\theta+\vartheta-y)\right\}} \\
& -\tau\left(\theta+\vartheta-\frac{x+y}{2}\right) \mid \\
& \quad \leq \frac{y-x}{4} \alpha^{\beta}\left\{\int_{0}^{1}\left(\frac{1-(1-\lambda)^{\alpha}}{\alpha}\right)^{\beta}\left|\tau^{\prime}\left(\theta+\vartheta-\left(\frac{2-\lambda}{2} x+\frac{\lambda}{2} y\right)\right)\right| d \lambda\right. \\
& \left.\quad+\int_{0}^{1}\left(\frac{1-(1-\lambda)^{\alpha}}{\alpha}\right)^{\beta}\left|\tau^{\prime}\left(\theta+\vartheta-\left(\frac{\lambda}{2} x+\frac{2-\lambda}{2} y\right)\right)\right| d \lambda\right\} \\
& \leq \frac{y-x}{4} \alpha^{\beta} \times\left\{\left(\left|\tau^{\prime}(\theta)\right|+\left|\tau^{\prime}(\vartheta)\right|\right) \int_{0}^{1}\left(\frac{1-(1-\lambda)^{\alpha}}{\alpha}\right)^{\beta} d \lambda\right. \\
& \left.-\left(\left|\tau^{\prime}(x)\right| \int_{0}^{1}\left(\frac{1-(1-\lambda)^{\alpha}}{\alpha}\right)^{\beta} \frac{2-\lambda}{2} d \lambda+\left|\tau^{\prime}(y)\right| \int_{0}^{1}\left(\frac{1-(1-\lambda)^{\alpha}}{\alpha}\right)^{\beta} \frac{\lambda}{2} d \lambda\right)\right\} \\
& +\left\{\left(\left|\tau^{\prime}(\theta)\right|+\left|\tau^{\prime}(\vartheta)\right|\right) \int_{0}^{1}\left(\frac{1-(1-\lambda)^{\alpha}}{\alpha}\right)^{\beta} d \lambda\right. \\
& \left.-\left(\left|\tau^{\prime}(x)\right| \int_{0}^{1}\left(\frac{1-(1-\lambda)^{\alpha}}{\alpha}\right)^{\beta} \frac{\lambda}{2} d \lambda+\left|\tau^{\prime}(y)\right| \int_{0}^{1}\left(\frac{1-(1-\lambda)^{\alpha}}{\alpha}\right)^{\beta} \frac{2-\lambda}{2} d \lambda\right)\right\} .
\end{aligned}
$$


By making use of the necessary computations, we get (2.29). Which completes the proof.

Remark 6. (i) If we take $x=\theta$ and $y=\vartheta$ in Theorem 4 , then we get (Theorem $3.1,[11])$.

(ii) If we take $\alpha=1, x=\theta$ and $y=\vartheta$ in Theorem 4 and $q=1$ in Theorem 5 of [25] are found to yield the same result.

Theorem 5. $\tau:[\theta, \vartheta] \rightarrow R$ is differentiable mapping on $(\theta, \vartheta)$ with $\theta<\vartheta$ and $\tau^{\prime} \in$ $L[\theta, \vartheta]$. If $\left|\tau^{\prime}\right|^{q}$ is convex function on $[\theta, \vartheta]$ for some fixed $q \geq 1$, then the following inequality holds for fractional integrals:

$$
\begin{aligned}
\mid \begin{array}{l}
2^{\alpha \beta-1} \alpha^{\beta} \Gamma(\beta+1) \\
(y-x)^{\alpha \beta}
\end{array}\left\{\begin{array}{l}
\beta \\
\left(\theta+\vartheta-\frac{x+y}{2}\right.
\end{array} J^{\alpha} \tau(\theta+\vartheta-x)+{ }^{\beta} J_{\left(\theta+\vartheta-\frac{x+y}{2}\right.}^{\alpha} \tau(\theta+\vartheta-y)\right\} \\
-\tau\left(\theta+\vartheta-\frac{x+y}{2}\right) \mid \\
\quad \leq \frac{y-x}{4} \alpha^{\beta}\left(\frac{1}{\alpha^{\beta+1}} B\left(\beta+1, \frac{1}{\alpha}\right)\right)^{1-\frac{1}{q}} \\
\quad\left\{\left(\left|\tau^{\prime}(\theta)\right|^{q}+\left|\tau^{\prime}(\vartheta)\right|^{q}\right)\left(\left(\frac{1}{\alpha^{\beta+1}} B\left(\beta+1, \frac{1}{\alpha}\right)\right)\right)\right. \\
-\left\{\left|\tau^{\prime}(x)\right|^{q}\left(\frac{1}{2 \alpha^{\beta+1}}\left(B\left(\beta+1, \frac{1}{\alpha}\right)-B\left(\beta+1, \frac{2}{\alpha}\right)\right)\right)\right. \\
\left.+\left|\tau^{\prime}(y)\right|^{q}\left(\frac{1}{2 \alpha^{\beta+1}}\left(B\left(\beta+1, \frac{2}{\alpha}\right)+B\left(\beta+1, \frac{1}{\alpha}\right)\right)\right)\right\} \\
+\left(\left|\tau^{\prime}(\theta)\right|^{q}+\left|\tau^{\prime}(\vartheta)\right|^{q}\right)\left(\left(\frac{1}{\alpha^{\beta+1}} B\left(\beta+1, \frac{1}{\alpha}\right)\right)\right) \\
-\left\{\left|\tau^{\prime}(x)\right|^{q}\left(\frac{1}{2 \alpha^{\beta+1}}\left(B\left(\beta+1, \frac{2}{\alpha}\right)-B\left(\beta+1, \frac{1}{\alpha}\right)\right)\right)\right. \\
\left.\left.+\left|\tau^{\prime}(y)\right|^{q}\left(\frac{1}{2 \alpha^{\beta+1}}\left(B\left(\beta+1, \frac{1}{\alpha}\right)+B\left(\beta+1, \frac{2}{\alpha}\right)\right)\right)\right\}\right\}^{\frac{1}{q}},
\end{aligned}
$$

where $\forall x, y \in[\theta, \vartheta], \alpha, \beta>0, \lambda \in[0,1]$ and $\Gamma(),. B(.,$.$) are the Euler Gamma$ and Beta function, $p^{-1}+q^{-1}=1$.

Proof. By using Lemma 1 with Jensen-Mercer inequality, by using convexity of $\left|\tau^{\prime}\right|^{q}$ and well-known power-mean inequality, we have 


$$
\begin{aligned}
& \mid \frac{2^{\alpha \beta-1} \alpha^{\beta} \Gamma(\beta+1)}{(y-x)^{\alpha \beta}}\left\{\begin{array}{l}
\beta \\
\left(\theta+\vartheta-\frac{x+y}{2}\right)
\end{array} J^{\alpha} \tau(\theta+\vartheta-x)+{ }^{\beta} J_{\left(\theta+\vartheta-\frac{x+y}{2}\right)}^{\alpha} \tau(\theta+\vartheta-y)\right\} \\
& -\tau\left(\theta+\vartheta-\frac{x+y}{2}\right) \\
& \leq \frac{y-x}{4} \alpha^{\beta}\left\{\left(\int_{0}^{1}\left(\frac{1-(1-\lambda)^{\alpha}}{\alpha}\right)^{\beta} d \lambda\right)^{1-\frac{1}{q}}\right. \\
& \times\left(\int_{0}^{1}\left(\frac{1-(1-\lambda)^{\alpha}}{\alpha}\right)^{\beta}\left|\tau^{\prime}\left(\theta+\vartheta-\left(\frac{\lambda}{2} x+\frac{2-\lambda}{2} y\right)\right)\right|^{q} d \lambda\right)^{\frac{1}{q}} \\
& +\left(\int_{0}^{1}\left(\frac{1-(1-\lambda)^{\alpha}}{\alpha}\right)^{\beta} d \lambda\right)^{1-\frac{1}{q}} \\
& \left.\times\left(\int_{0}^{1}\left(\frac{1-(1-\lambda)^{\alpha}}{\alpha}\right)^{\beta}\left|\tau^{\prime}\left(\theta+\vartheta-\left(\frac{2-\lambda}{2} x+\frac{\lambda}{2} y\right)\right)\right|^{q} d \lambda\right)^{\frac{1}{q}}\right\} \\
& \leq \frac{y-x}{4} \alpha^{\beta}\left\{\left(\int_{0}^{1}\left(\frac{1-(1-\lambda)^{\alpha}}{\alpha}\right)^{\beta} d \lambda\right)^{1-\frac{1}{q}}\right. \\
& \times\left(\int_{0}^{1}\left(\frac{1-(1-\lambda)^{\alpha}}{\alpha}\right)^{\beta}\right. \\
& \left.\left(\left(\left|\tau^{\prime}(\theta)\right|^{q}+\left|\tau^{\prime}(\vartheta)\right|^{q}\right)-\left(\frac{\lambda}{2}\left|\tau^{\prime}(x)\right|^{q}+\frac{2-\lambda}{2}\left|\tau^{\prime}(y)\right|^{q}\right)\right) d \lambda\right)^{\frac{1}{q}} \\
& +\left(\int_{0}^{1}\left(\frac{1-(1-\lambda)^{\alpha}}{\alpha}\right)^{\beta} d \lambda\right)^{1-\frac{1}{q}} \\
& \times\left(\int_{0}^{1}\left(\frac{1-(1-\lambda)^{\alpha}}{\alpha}\right)^{\beta}\right. \\
& \left.\left.\left(\left(\left|\tau^{\prime}(\theta)\right|^{q}+\left|\tau^{\prime}(\vartheta)\right|^{q}\right)-\left(\frac{2-\lambda}{2}\left|\tau^{\prime}(x)\right|^{q}+\frac{\lambda}{2}\left|\tau^{\prime}(y)\right|^{q}\right)\right) d \lambda\right)^{\frac{1}{q}}\right\} .
\end{aligned}
$$

Making necessary simplifications, we get (2.30). The proof is completed.

Remark 7. (i) If we take $x=\theta$ and $y=\vartheta$ in Theorem 5 , then we get (Theorem $3.2,[11])$.

(ii) If we take $\alpha=1, x=\theta$ and $y=\vartheta$ in Theorem 5 reduces to Theorem 5 in [25]. 
Theorem 6. $\tau:[\theta, \vartheta] \rightarrow R$ is differentiable mapping on $(\theta, \vartheta)$ with $\theta<\vartheta$ and $\tau^{\prime} \in L[\theta, \vartheta]$. If $\left|\tau^{\prime}\right|^{q}$ is convex function on $[\theta, \vartheta]$, then the following inequality holds for fractional integrals:

$$
\begin{gathered}
\left.\mid \frac{2^{\alpha \beta-1} \alpha^{\beta} \Gamma(\beta+1)}{(y-x)^{\alpha \beta}}\left\{\begin{array}{l}
\beta \\
\left(\theta+\vartheta-\frac{x+y}{2}\right.
\end{array}\right) J^{\alpha} \tau(\theta+\vartheta-x)+{ }^{\beta} J_{\left(\theta+\vartheta-\frac{x+y}{2}\right.}^{\alpha} \tau(\theta+\vartheta-y)\right\} \\
-\tau\left(\theta+\vartheta-\frac{x+y}{2}\right) \mid \\
\leq \frac{y-x}{4} \alpha^{\beta}\left(\frac{1}{\alpha^{\beta p+1}} B\left(\beta p+1, \frac{1}{\alpha}\right)\right)^{\frac{1}{p}} \\
\times\left\{\left(\left|\tau^{\prime}(\theta)\right|^{q}+\left|\tau^{\prime}(\vartheta)\right|^{q}-\left(\frac{3\left|\tau^{\prime}(x)\right|^{q}+\left|\tau^{\prime}(y)\right|^{q}}{4}\right)\right)^{\frac{1}{q}}\right. \\
\left.+\left(\left|\tau^{\prime}(\theta)\right|^{q}+\left|\tau^{\prime}(\vartheta)\right|^{q}-\left(\frac{\left|\tau^{\prime}(x)\right|^{q}+3\left|\tau^{\prime}(y)\right|^{q}}{4}\right)\right)^{\frac{1}{q}}\right\},
\end{gathered}
$$

where $\forall x, y \in[\theta, \vartheta], \alpha, \beta>0, \lambda \in[0,1], q>0$ and $\Gamma(),. B(.,$.$) are the Euler$ Gamma and Beta function, $p^{-1}+q^{-1}=1$.

Proof. If we proceed a similar argument to the proof of the previous theorem, by using Lemma 1 with Jensen-Mercer inequality and familiar Hölder integral inequality, we can write

$$
\begin{aligned}
& \mid \frac{2^{\alpha \beta-1} \alpha^{\beta} \Gamma(\beta+1)}{(y-x)^{\alpha \beta}}\left\{\begin{array}{l}
\beta \\
\left(\theta+\vartheta-\frac{x+y}{2}\right)
\end{array} J^{\alpha} \tau(\theta+\vartheta-x)+{ }^{\beta} J_{\left(\theta+\vartheta-\frac{x+y}{2}\right)}^{\alpha} \tau(\theta+\vartheta-y)\right\} \\
& -\tau\left(\theta+\vartheta-\frac{x+y}{2}\right) \mid \\
& \leq \frac{y-x}{4} \alpha^{\beta}\left(\int_{0}^{1}\left(\frac{1-(1-\lambda)^{\alpha}}{\alpha}\right){ }^{\beta p} d t\right) \\
& \times\left\{\left(\int_{0}^{1}\left|\tau^{\prime}\left(\theta+\vartheta-\left(\frac{2-\lambda}{2} x+\frac{\lambda}{2} y\right)\right)\right|^{\frac{1}{p}}\right)^{\frac{1}{q}} d \lambda\right. \\
& \left.+\left(\int_{0}^{1}\left|\tau^{\prime}\left(\theta+\vartheta-\left(\frac{\lambda}{2} x+\frac{2-\lambda}{2} y\right)\right)\right|^{q}\right)^{\frac{1}{q}} d \lambda\right\} .
\end{aligned}
$$


By applying the convexity of $\left|\tau^{\prime}\right|^{q}$, we have

$$
\begin{gathered}
\left|\tau^{\prime}\left(\theta+\vartheta-\left(\frac{2-\lambda}{2} x+\frac{\lambda}{2} y\right)\right)\right|^{q} \\
\leq\left|\tau^{\prime}(\theta)\right|^{q}+\left|\tau^{\prime}(\vartheta)\right|^{q}-\left(\frac{2-\lambda}{2}\left|\tau^{\prime}(x)\right|^{q}+\frac{\lambda}{2}\left|\tau^{\prime}(y)\right|^{q}\right) \\
\leq \frac{y-x}{4} \alpha^{\beta}\left(\int_{0}^{1}\left(\frac{1-(1-\lambda)^{\alpha}}{\alpha}\right)^{\beta p} d \lambda\right)^{\frac{1}{p}} \\
\times\left\{\left(\int_{0}^{1}\left(\left|\tau^{\prime}(\theta)\right|^{q}+\left|\tau^{\prime}(\vartheta)\right|^{q}-\left(\frac{2-\lambda}{2}\left|\tau^{\prime}(x)\right|^{q}+\frac{\lambda}{2}\left|\tau^{\prime}(y)\right|^{q}\right)\right)\right)^{\frac{1}{q}} d \lambda\right. \\
\left.+\left(\int_{0}^{1}\left(\left|\tau^{\prime}(\theta)\right|^{q}+\left|\tau^{\prime}(\vartheta)\right|^{q}-\left(\frac{\lambda}{2}\left|\tau^{\prime}(x)\right|^{q}+\frac{2-\lambda}{2}\left|\tau^{\prime}(y)\right|^{q}\right)\right)\right)^{\frac{1}{q}} d \lambda\right\} \\
\left.\leq \frac{y-x}{4} \alpha^{\beta}\left(\frac{1}{\alpha^{\beta p+1}} B\left(\beta p+1, \frac{1}{\alpha}\right)\right)^{\frac{1}{p}}\right\} \\
\times\left\{\left(\left|\tau^{\prime}(\theta)\right|^{q}+\left|\tau^{\prime}(\vartheta)\right|^{q}-\left(\frac{3\left|\tau^{\prime}(x)\right|^{q}+\left|\tau^{\prime}(y)\right|^{q}}{4}\right)\right)^{\frac{1}{q}}\right. \\
\left.+\left(\left|\tau^{\prime}(\theta)\right|^{q}+\left|\tau^{\prime}(\vartheta)\right|^{q}-\left(\frac{\left|\tau^{\prime}(x)\right|^{q}+3\left|\tau^{\prime}(y)\right|^{q}}{4}\right)\right)^{\frac{1}{q}}\right\},
\end{gathered}
$$

which completes the proof.

Corollary 2. If we choose $\alpha=1$ in Theorem 6 , then we have the following inequality

$$
\begin{aligned}
& \left|\frac{1}{y-x} \int_{\theta+\vartheta-y}^{\theta+\vartheta-x} \tau(\lambda) d \lambda-\tau\left(\theta+\vartheta-\frac{x+y}{2}\right)\right| \\
& \leq \frac{1}{2^{\frac{1}{p}}} \times\left\{\left(\left|\tau^{\prime}(\theta)\right|^{q}+\left|\tau^{\prime}(\vartheta)\right|^{q}-\left(\frac{3\left|\tau^{\prime}(x)\right|^{q}+\left|\tau^{\prime}(y)\right|^{q}}{4}\right)\right)^{\frac{1}{q}}\right. \\
& \left.+\left(\left|\tau^{\prime}(\theta)\right|^{q}+\left|\tau^{\prime}(\vartheta)\right|^{q}-\left(\frac{\left|\tau^{\prime}(x)\right|^{q}+3\left|\tau^{\prime}(y)\right|^{q}}{4}\right)\right)^{\frac{1}{q}}\right\} .
\end{aligned}
$$

Theorem 7. $\tau:[\theta, \vartheta] \rightarrow R$ is differentiable mapping on $(\theta, \vartheta)$ with $\theta<\vartheta$ and $\tau^{\prime} \in L[\theta, \vartheta]$. If $\left|\tau^{\prime}\right|^{q}$ is convex function on $[\theta, \vartheta], q>1$, then the following inequality 
holds for fractional integrals:

$$
\begin{aligned}
& \mid \frac{2^{\alpha \beta-1} \alpha^{\beta} \Gamma(\beta+1)}{(y-x)^{\alpha \beta}\left\{\begin{array}{l}
\beta \\
\left(\theta+\vartheta-\frac{x+y}{2}\right.
\end{array} J^{\alpha} \tau(\theta+\vartheta-x)+{ }^{\beta} J_{\left(\theta+\vartheta-\frac{x+y}{2}\right.}^{\alpha} \tau(\theta+\vartheta-y)\right\}} \\
& -\tau\left(\theta+\vartheta-\frac{x+y}{2}\right) \mid \\
& \leq \frac{(y-x) \alpha^{\beta}}{4}\left\{\left(\left|\tau^{\prime}(\theta)\right|^{q}+\left|\tau^{\prime}(\vartheta)\right|^{q}\right)\left(\frac{B\left(\beta+1, \frac{1}{\alpha}\right)}{\alpha^{\beta+1}}\right)\right. \\
& -\left(\left(\frac{B\left(\beta+1, \frac{2}{\alpha}\right)+B\left(\beta+1, \frac{1}{\alpha}\right)}{2 \alpha^{\beta+1}}\right)\left|\tau^{\prime}(x)\right|^{q}\right. \\
& \left.+\left(\frac{B\left(\beta+1, \frac{1}{\alpha}\right)-B\left(\beta+1, \frac{2}{\alpha}\right)}{2 \alpha^{\beta+1}}\right)\left|\tau^{\prime}(y)\right|^{q}\right)^{\frac{1}{q}} \\
& +\left(\left|\tau^{\prime}(\theta)\right|^{q}+\left|\tau^{\prime}(\vartheta)\right|^{q}\right)\left(\frac{B\left(\beta+1, \frac{1}{\alpha}\right)}{\alpha^{\beta+1}}\right) \\
& -\left(\left(\frac{B\left(\beta+1, \frac{1}{\alpha}\right)-B\left(\beta+1, \frac{2}{\alpha}\right)}{2 \alpha^{\beta+1}}\right)\left|\tau^{\prime}(x)\right|^{q}\right. \\
& \left.\left.+\left(\frac{B\left(\beta+1, \frac{2}{\alpha}\right)+B\left(\beta+1, \frac{1}{\alpha}\right)}{2 \alpha^{\beta+1}}\right)\left|\tau^{\prime}(y)\right|^{q}\right)^{\frac{1}{q}}\right\},
\end{aligned}
$$

where ll $x, y \in[\theta, \vartheta], \alpha, \beta>0, \lambda \in[0,1]$ and $\Gamma(),. B(.,$.$) are the Euler Gamma$ and Beta function, $p^{-1}+q^{-1}=1$.

Proof. By using Lemma 1 with Jensen-Mercer inequality, convexity of $\left|\tau^{\prime}\right|^{q}$ and familiar Hölder integral inequality, we obtain

$$
\begin{aligned}
& \mid \frac{2^{\alpha \beta-1} \alpha^{\beta} \Gamma(\beta+1)}{(y-x)^{\alpha \beta}}\left\{\begin{array}{l}
\beta \\
\left(\theta+\vartheta-\frac{x+y}{2}\right)
\end{array} J^{\alpha} \tau(\theta+\vartheta-x)+\beta J_{\left(\theta+\vartheta-\frac{x+y}{2}\right)}^{\alpha} \tau(\theta+\vartheta-y)\right\} \\
& -\tau\left(a+b-\frac{x+y}{2}\right) \mid
\end{aligned}
$$




$$
\begin{aligned}
& \leq \frac{(y-x) \alpha^{\beta}}{4}\left\{\left(\int_{0}^{1} 1 d \lambda\right)^{\frac{1}{p}}\right. \\
& \times\left(\int_{0}^{1}\left(\frac{1-(1-\lambda)^{\alpha}}{\alpha}\right)^{\beta}\left|\tau^{\prime}\left(\theta+\vartheta-\left(\frac{2-\lambda}{2} x+\frac{\lambda}{2} y\right)\right)\right|^{q} d \lambda\right)^{\frac{1}{q}} \\
& +\left(\int_{0}^{1} 1 d \lambda\right)^{\frac{1}{p}} \\
& \left.\times\left(\int_{0}^{1}\left(\frac{1-(1-\lambda)^{\alpha}}{\alpha}\right)^{\beta}\left|\tau^{\prime}\left(\theta+\vartheta-\left(\frac{\lambda}{2} x+\frac{2-\lambda}{2} y\right)\right)\right|^{q} d \lambda\right)^{\frac{1}{q}}\right\} .
\end{aligned}
$$

By making necessary changes, we get (2.33).

Theorem 8. $\tau:[\theta, \vartheta] \rightarrow R$ is differentiable mapping on $(\theta, \vartheta)$ with $\theta<\vartheta$ and $\tau^{\prime} \in L[\theta, \vartheta]$. If $\left|\tau^{\prime}\right|$ is convex function on $[\theta, \vartheta]$, then the following inequality holds for fractional integrals:

$$
\begin{aligned}
& \quad \mid \frac{\tau(\theta+\vartheta-x)+\tau(\theta+\vartheta-y)}{2}-\frac{\alpha^{\beta} \Gamma(\beta+1)}{2(y-x)^{\alpha \beta}} \\
& \quad \times\left\{\begin{array}{l}
\beta \\
(\theta+\vartheta-y)
\end{array} J^{\alpha} \tau(\theta+\vartheta-x)+{ }^{\beta} J_{(\theta+\vartheta-x)}^{\alpha} \tau(\theta+\vartheta-y)\right\} \mid \\
& \leq \frac{(y-x) \alpha^{\beta}}{2}\left[\left\{\left(\left|\tau^{\prime}(\theta)\right|+\left|\tau^{\prime}(\vartheta)\right|\right) \frac{B\left(\frac{1}{\alpha}, \beta+1\right)}{\alpha^{\beta+1}}\right.\right. \\
& -\frac{\left|\tau^{\prime}(x)\right|}{\alpha^{\beta+1}}\left\{B_{\frac{1}{2^{\alpha}}}\left(\frac{1}{\alpha}, \beta+1\right)+B\left(\frac{2}{\alpha}, \beta+1\right)-B\left(\frac{1}{\alpha}, \beta+1\right)\right\} \\
& \left.-\frac{\left|\tau^{\prime}(y)\right|}{\alpha^{\beta+1}}\left\{B_{\frac{1}{2^{\alpha}}}\left(\frac{1}{\alpha}, \beta+1\right)-B\left(\frac{2}{\alpha}, \beta+1\right)\right\}\right\} \\
& +\left\{\left(\left|\tau^{\prime}(\theta)\right|+\left|\tau^{\prime}(\vartheta)\right|\right) \frac{B\left(\frac{1}{\alpha}, \beta+1\right)}{\alpha^{\beta+1}}\right. \\
& -\frac{\left|\tau^{\prime}(x)\right|}{\alpha^{\beta+1}}\left\{B_{\frac{1}{2^{\alpha}}}\left(\frac{1}{\alpha}, \beta+1\right)-B\left(\frac{2}{\alpha}, \beta+1\right)\right\} \\
& \left.\left.-\frac{\left|\tau^{\prime}(y)\right|}{\alpha^{\beta+1}}\left\{B_{\frac{1}{2^{\alpha}}}\left(\frac{1}{\alpha}, \beta+1\right)+B\left(\frac{2}{\alpha}, \beta+1\right)-B\left(\frac{1}{\alpha}, \beta+1\right)\right\}\right\}\right]
\end{aligned}
$$

where $\forall x, y \in[\theta, \vartheta], \alpha, \beta>0, \lambda \in[0,1]$ and $\Gamma(),. B(.,$.$) are the Euler Gamma$ and Beta function. 
Proof. By a similar way with the proof the previous theorem, we get

$$
\begin{aligned}
& \mid \frac{\tau(\theta+\vartheta-x)+\tau(\theta+\vartheta-y)}{2}-\frac{\alpha^{\beta} \Gamma(\beta+1)}{2(y-x)^{\alpha \beta}} \\
& \times\left\{\begin{array}{l}
\beta \\
(\theta+\vartheta-y)
\end{array} J^{\alpha} \tau(\theta+\vartheta-x)+{ }^{\beta} J_{(\theta+\vartheta-x)}^{\alpha} \tau(\theta+\vartheta-y)\right\} \mid \\
& \leq \frac{(y-x) \alpha^{\beta}}{2} \int_{0}^{1}\left|\left(\frac{1-(1-\lambda)^{\alpha}}{\alpha}\right)^{\beta}-\left(\frac{1-\lambda^{\alpha}}{\alpha}\right)^{\beta}\right|\left|\tau^{\prime}(\theta+\vartheta-(\lambda x+(1-\lambda) y))\right| d \lambda \\
& \leq \frac{(y-x) \alpha^{\beta}}{2} \int_{0}^{1}\left|\left(\frac{1-(1-\lambda)^{\alpha}}{\alpha}\right)^{\beta}-\left(\frac{1-\lambda^{\alpha}}{\alpha}\right)^{\beta}\right| \\
& \times\left\{\left|\tau^{\prime}(\theta)\right|+\left|\tau^{\prime}(\vartheta)\right|-\left(\lambda\left|\tau^{\prime}(x)\right|+(1-\lambda)\left|\tau^{\prime}(y)\right|\right)\right\} d \lambda \\
& \leq \frac{(y-x) \alpha^{\beta}}{2}\left[\int_{0}^{\frac{1}{2}}\left[\left(\frac{1-\lambda{ }^{\alpha}}{\alpha}\right)^{\beta}-\left(\frac{1-(1-\lambda)^{\alpha}}{\alpha}\right)^{\beta}\right]\right. \\
& \times\left\{\left|\tau^{\prime}(\theta)\right|+\left|\tau^{\prime}(\vartheta)\right|-\left(\lambda\left|\tau^{\prime}(x)\right|+(1-\lambda)\left|\tau^{\prime}(y)\right|\right)\right\} d \lambda \\
& +\int_{\frac{1}{2}}^{1}\left[\left(\frac{1-(1-\lambda)^{\alpha}}{\alpha}\right)^{\beta}-\left(\frac{1-\lambda}{\alpha}\right)^{\beta}\right] \\
& \left.\times\left\{\left|\tau^{\prime}(\theta)\right|+\left|\tau^{\prime}(\vartheta)\right|-\left(\lambda\left|\tau^{\prime}(x)\right|+(1-\lambda)\left|\tau^{\prime}(y)\right|\right)\right\} d \lambda\right] .
\end{aligned}
$$

This completes the proof.

\section{NEW INEQUALITIES VIA IMPROVED HöLDER INEQUALITY}

Theorem 9. $\tau:[\theta, \vartheta] \rightarrow R$ is differentiable mapping on $(\theta, \vartheta)$ with $\theta<\vartheta$ and $\tau^{\prime} \in L[\theta, \vartheta]$. If $\left|\tau^{\prime}\right|^{q}$ is convex function on $[\theta, \vartheta], q>1$, then the following inequality holds for fractional integrals:

$$
\begin{aligned}
& \mid \frac{2^{\alpha \beta-1} \alpha^{\beta} \Gamma(\beta+1)}{(y-x)^{\alpha \beta}}\left\{\begin{array}{l}
\beta \\
\left(\theta+\vartheta-\frac{x+y}{2}\right)
\end{array} J^{\alpha} \tau(\theta+\vartheta-x)+\beta J_{\left(\theta+\vartheta-\frac{x+y}{2}\right)}^{\alpha} \tau(\theta+\vartheta-y)\right\} \\
& -\tau\left(\theta+\vartheta-\frac{x+y}{2}\right) \mid
\end{aligned}
$$




$$
\begin{aligned}
& \leq \frac{(y-x) \alpha^{\beta}}{2}\left[\left\{\left(\frac{B\left(\frac{2}{\alpha}, \beta p+1\right)}{\alpha^{\beta p+1}}\right)^{\frac{1}{p}}\right.\right. \\
& \times\left(\frac{\left|\tau^{\prime}(\theta)\right|^{q}+\left|\tau^{\prime}(\vartheta)\right|^{q}}{2}-\left(\frac{5}{12}\left|\tau^{\prime}(x)\right|^{q}+\frac{1}{12}\left|\tau^{\prime}(y)\right|^{q}\right)\right)^{\frac{1}{q}} \\
& +\left(\frac{B\left(\frac{1}{\alpha}, \beta p+1\right)-B\left(\frac{2}{\alpha}, \beta p+1\right)}{\alpha^{\beta p+1}}\right)^{\frac{1}{p}} \\
& \left.\times\left(\frac{\left|\tau^{\prime}(\theta)\right|^{q}+\left|\tau^{\prime}(\vartheta)\right|^{q}}{2}-\left(\frac{1}{3}\left|\tau^{\prime}(x)\right|^{q}+\frac{1}{6}\left|\tau^{\prime}(y)\right|^{q}\right)\right)^{\frac{1}{q}}\right\} \\
& +\left\{\left(\frac{B\left(\frac{2}{\alpha}, \beta p+1\right)}{\alpha^{\beta p+1}}\right)^{\frac{1}{p}},\right. \\
& \times\left(\frac{\left|\tau^{\prime}(\theta)\right|^{q}+\left|\tau^{\prime}(\vartheta)\right|^{q}}{2}-\left(\frac{1}{12}\left|\tau^{\prime}(x)\right|^{q}+\frac{5}{12}\left|\tau^{\prime}(y)\right|^{q}\right)\right)^{\frac{1}{q}} \\
& +\left(\frac{B\left(\frac{1}{\alpha}, \beta p+1\right)-B\left(\frac{2}{\alpha}, \beta p+1\right)}{\alpha^{\beta p+1}}\right)^{\frac{1}{p}} \\
& \left.\left.\times\left(\frac{\left|\tau^{\prime}(\theta)\right|^{q}+\left|\tau^{\prime}(\vartheta)\right|^{q}}{2}-\left(\frac{1}{6}\left|\tau^{\prime}(x)\right|^{q}+\frac{1}{3}\left|\tau^{\prime}(y)\right|^{q}\right)\right)^{\frac{1}{q}}\right\}\right],
\end{aligned}
$$

where $\forall x, y \in[\theta, \vartheta], \alpha, \beta>0, \lambda \in[0,1]$ and $\Gamma(),. B(.,$.$) are the Euler Gamma$ and Beta function, $p^{-1}+q^{-1}=1$.

Proof. By using Lemma 1 with Jensen-Mercer inequality, convexity of $\left|\tau^{\prime}\right|^{q}$ and applying the Hölder-İ̧can integral inequality that is given in (Theorem 1.4, [21]), we can write

$$
\begin{aligned}
& \mid \frac{2^{\alpha \beta-1} \alpha^{\beta} \Gamma(\beta+1)}{(y-x)^{\alpha \beta}}\left\{\begin{array}{l}
\beta \\
\left(\theta+\vartheta-\frac{x+y}{2}\right)
\end{array} J^{\alpha} \tau(\theta+\vartheta-x)+{ }^{\beta} J_{\left(\theta+\vartheta-\frac{x+y}{2}\right)}^{\alpha} \tau(\theta+\vartheta-y)\right\} \\
& -\tau\left(a+b-\frac{x+y}{2}\right) \mid
\end{aligned}
$$




$$
\begin{aligned}
& \leq \frac{(y-x) \alpha^{\beta}}{4}\left[\left\{\left(\int_{0}^{1}(1-\lambda)\left(\frac{1-(1-\lambda)^{\alpha}}{\alpha}\right)^{\beta p} d \lambda\right)^{\frac{1}{p}}\right.\right. \\
& \times\left(\int_{0}^{1}(1-\lambda)\left[\left|\tau^{\prime}(\theta)\right|^{q}+\left|\tau^{\prime}(\vartheta)\right|^{q}-\left(\frac{2-\lambda}{2}\left|\tau^{\prime}(x)\right|^{q}+\frac{\lambda}{2}\left|\tau^{\prime}(y)\right|^{q}\right)\right] d \lambda\right)^{\frac{1}{q}} \\
& \left(\int_{0}^{1} \lambda\left(\frac{1-(1-\lambda)^{\alpha}}{\alpha}\right)^{\beta p} d \lambda\right)^{\frac{1}{p}} \\
& \left.\times\left(\int_{0}^{1} \lambda\left[\left|\tau^{\prime}(\theta)\right|^{q}+\left|\tau^{\prime}(\vartheta)\right|^{q}-\left(\frac{2-\lambda}{2}\left|\tau^{\prime}(x)\right|^{q}+\frac{\lambda}{2}\left|\tau^{\prime}(y)\right|^{q}\right)\right] d \lambda\right)^{\frac{1}{q}}\right\} \\
& +\left\{\left(\int_{0}^{1}(1-\lambda)\left(\frac{1-(1-\lambda)^{\alpha}}{\alpha}\right)^{\beta p} d \lambda\right)^{\frac{1}{p}}\right. \\
& \times\left(\int_{0}^{1}(1-\lambda)\left[\left|\tau^{\prime}(\theta)\right|^{q}+\left|\tau^{\prime}(\vartheta)\right|^{q}-\left(\frac{\lambda}{2}\left|\tau^{\prime}(x)\right|^{q}+\frac{2-\lambda}{2}\left|\tau^{\prime}(y)\right|^{q}\right)\right] d \lambda\right)^{\frac{1}{q}} \\
& \left(\int_{0}^{1} \lambda\left(\frac{1-(1-\lambda)^{\alpha}}{\alpha}\right)^{\beta p} d \lambda\right)^{\frac{1}{p}} \\
& \left.\left.\times\left(\int_{0}^{1} \lambda\left[\left|\tau^{\prime}(\theta)\right|^{q}+\left|\tau^{\prime}(\vartheta)\right|^{q}-\left(\frac{\lambda}{2}\left|\tau^{\prime}(x)\right|^{q}+\frac{2-\lambda}{2}\left|\tau^{\prime}(y)\right|^{q}\right)\right] d \lambda\right)^{\frac{1}{q}}\right\}\right] .
\end{aligned}
$$

By using calculus tools, one can have the required result.

Theorem 10. $\tau:[\theta, \vartheta] \rightarrow R$ is differentiable mapping on $(\theta, \vartheta)$ with $\theta<\vartheta$ and $\tau^{\prime} \in L[\theta, \vartheta]$. If $\left|\tau^{\prime}\right|^{q}$ is convex function on $[\theta, \vartheta]$, for some $q \geq 1$, then the following inequality holds for fractional integrals:

$$
\begin{aligned}
& \mid \frac{2^{\alpha \beta-1} \alpha^{\beta} \Gamma(\beta+1)}{(y-x)^{\alpha \beta}}\left\{\begin{array}{l}
\beta \\
\left(\theta+\vartheta-\frac{x+y}{2}\right)
\end{array} J^{\alpha} \tau(\theta+\vartheta-x)+\beta J_{\left(\theta+\vartheta-\frac{x+y}{2}\right)}^{\alpha} \tau(\theta+\vartheta-y)\right\} \\
& -\tau\left(\theta+\vartheta-\frac{x+y}{2}\right) \mid
\end{aligned}
$$




$$
\begin{aligned}
& \leq \frac{(y-x) \alpha^{\beta}}{4}\left[\left\{\left(\frac{B\left(\frac{2}{\alpha}, \beta+1\right)}{\alpha^{\beta+1}}\right)^{1-\frac{1}{q}}\right.\right. \\
& \times\left(\left(\left|\tau^{\prime}(\theta)\right|^{q}+\left|\tau^{\prime}(\vartheta)\right|^{q}\right)\left(\frac{B\left(\frac{2}{\alpha}, \beta+1\right)}{\alpha^{\beta+1}}\right)\right. \\
& -\left(\frac{1}{2 \alpha^{\beta+1}}\left|\tau^{\prime}(x)\right|^{q}\left(B\left(\frac{2}{\alpha}, \beta+1\right)+B\left(\frac{3}{\alpha}, \beta+1\right)\right)\right. \\
& \left.\left.+\frac{1}{2 \alpha^{\beta+1}}\left|\tau^{\prime}(y)\right|^{q}\left(B\left(\frac{2}{\alpha}, \beta+1\right)-B\left(\frac{3}{\alpha}, \beta+1\right)\right)\right)\right)^{\frac{1}{q}} \\
& +\left(\frac{B\left(\frac{1}{\alpha}, \beta+1\right)-B\left(\frac{2}{\alpha}, \beta+1\right)}{\alpha^{\beta+1}}\right)^{1-\frac{1}{q}} \\
& \times\left(\left(\left|\tau^{\prime}(\theta)\right|^{q}+\left|\tau^{\prime}(\vartheta)\right|^{q}\right)\left(\frac{B\left(\frac{1}{\alpha}, \beta+1\right)-B\left(\frac{2}{\alpha}, \beta+1\right)}{\alpha^{\beta+1}}\right)\right. \\
& -\left(\frac{1}{2 \alpha^{\beta+1}}\left|\tau^{\prime}(x)\right|^{q}\left(B\left(\frac{1}{\alpha}, \beta+1\right)-B\left(\frac{3}{\alpha}, \beta+1\right)\right)\right. \\
& +\frac{1}{2 \alpha^{\beta+1}}\left|\tau^{\prime}(y)\right|^{q}\left(B\left(\frac{1}{\alpha}, \beta+1\right)-2 B\left(\frac{2}{\alpha}, \beta+1\right)\right. \\
& \left.\left.\left.\left.+B\left(\frac{3}{\alpha}, \beta+1\right)\right)\right)\right)^{\frac{1}{q}}\right\} \\
& +\left\{( \frac { B ( \frac { 2 } { \alpha } , \beta + 1 ) } { \alpha ^ { \beta + 1 } } ) ^ { 1 - \frac { 1 } { q } } \left(\left(\left|\tau^{\prime}(\theta)\right|^{q}+\left|\tau^{\prime}(\vartheta)\right|^{q}\right)\left(\frac{B\left(\frac{2}{\alpha}, \beta+1\right)}{\alpha^{\beta+1}}\right)\right.\right. \\
& -\left(\frac{1}{2 \alpha^{\beta+1}}\left|\tau^{\prime}(x)\right|^{q}\left(B\left(\frac{2}{\alpha}, \beta+1\right)-B\left(\frac{3}{\alpha}, \beta+1\right)\right)\right. \\
& \left.\left.+\frac{1}{2 \alpha^{\beta+1}}\left|\tau^{\prime}(y)\right|^{q}\left(B\left(\frac{2}{\alpha}, \beta+1\right)+B\left(\frac{3}{\alpha}, \beta+1\right)\right)\right)\right)^{\frac{1}{q}} \\
& +\left(\frac{B\left(\frac{1}{\alpha}, \beta+1\right)-B\left(\frac{2}{\alpha}, \beta+1\right)}{\alpha^{\beta+1}}\right)^{1-\frac{1}{q}} \\
& \left(\left(\left|\tau^{\prime}(\theta)\right|^{q}+\left|\tau^{\prime}(\vartheta)\right|^{q}\right)\left(\frac{B\left(\frac{1}{\alpha}, \beta+1\right)-B\left(\frac{2}{\alpha}, \beta+1\right)}{\alpha^{\beta+1}}\right)\right. \\
& -\left(\frac{1}{2 \alpha^{\beta+1}}\left|\tau^{\prime}(x)\right|^{q}\left(B\left(\frac{1}{\alpha}, \beta+1\right)-2 B\left(\frac{2}{\alpha}, \beta+1\right)+B\left(\frac{3}{\alpha}, \beta+1\right)\right)\right. \\
& \left.\left.\left.\left.+\frac{1}{2 \alpha^{\beta+1}}\left|\tau^{\prime}(y)\right|^{q}\left(B\left(\frac{1}{\alpha}, \beta+1\right)-B\left(\frac{3}{\alpha}, \beta+1\right)\right)\right)\right)^{\frac{1}{q}}\right\}\right],
\end{aligned}
$$


where $\forall x, y \in[\theta, \vartheta], \alpha, \beta>0, \lambda \in[0,1]$ and $\Gamma(),. B(.,$.$) are the Euler Gamma$ and Beta function, $p^{-1}+q^{-1}=1$.

Proof. By using Lemma 1 with Jensen-Mercer inequality, convexity of $\left|\tau^{\prime}\right|^{q}$ and applying the Improved power-mean integral inequality (Theorem 1.5, [21]), we have

$$
\begin{aligned}
& \mid \frac{2^{\alpha \beta-1} \alpha^{\beta} \Gamma(\beta+1)}{(y-x)^{\alpha \beta}}\left\{\begin{array}{l}
\beta \\
\left(\theta+\vartheta-\frac{x+y}{2}\right)
\end{array} J^{\alpha} \tau(\theta+\vartheta-x)+{ }^{\beta} J_{\left(\theta+\vartheta-\frac{x+y}{2}\right.}^{\alpha} \tau(\theta+\vartheta-y)\right\} \\
& -\tau\left(\theta+\vartheta-\frac{x+y}{2}\right) \\
& \leq \frac{(y-x) \alpha^{\beta}}{4}\left[\left\{\left(\int_{0}^{1}(1-\lambda)\left(\frac{1-(1-\lambda)^{\alpha}}{\alpha}\right)^{\beta} d \lambda\right)^{1-\frac{1}{q}}\right.\right. \\
& \times\left(\int_{0}^{1}(1-\lambda)\left(\frac{1-(1-\lambda)^{\alpha}}{\alpha}\right)^{\beta}\left|\tau^{\prime}\left(\theta+\vartheta-\left(\frac{2-\lambda}{2} x+\frac{\lambda}{2} y\right)\right)\right|^{q} d \lambda\right)^{\frac{1}{q}} \\
& +\left(\int_{0}^{1} \lambda\left(\frac{1-(1-\lambda)^{\alpha}}{\alpha}\right)^{\beta} d \lambda\right)^{1-\frac{1}{q}} \\
& \left.\times\left(\int_{0}^{1} \lambda\left(\frac{1-(1-\lambda)^{\alpha}}{\alpha}\right)^{\beta}\left|\tau^{\prime}\left(\theta+\vartheta-\left(\frac{2-\lambda}{2} x+\frac{\lambda}{2} y\right)\right)\right|^{q} d \lambda\right)^{\frac{1}{q}}\right\} \\
& +\left\{\left(\int_{0}^{1}(1-\lambda)\left(\frac{1-(1-\lambda)^{\alpha}}{\alpha}\right)^{\beta} d \lambda\right)^{1-\frac{1}{q}}\right. \\
& \times\left(\int_{0}^{1}(1-\lambda)\left(\frac{1-(1-\lambda)^{\alpha}}{\alpha}\right)^{\beta}\left|\tau^{\prime}\left(\theta+\vartheta-\left(\frac{\lambda}{2} x+\frac{2-\lambda}{2} y\right)\right)\right|^{q} d \lambda\right)^{\frac{1}{q}} \\
& +\left(\int_{0}^{1} \lambda\left(\frac{1-(1-\lambda)^{\alpha}}{\alpha}\right)^{\beta} d \lambda\right)^{1-\frac{1}{q}} \\
& \left.\left.\times\left(\int_{0}^{1} \lambda\left(\frac{1-(1-\lambda)^{\alpha}}{\alpha}\right)^{\beta}\left|\tau^{\prime}\left(\theta+\vartheta-\left(\frac{\lambda}{2} x+\frac{2-\lambda}{2} y\right)\right)\right|^{q} d \lambda\right)^{\frac{1}{q}}\right\}\right]
\end{aligned}
$$

By computing the above integrals, one can have the required result.

\section{ACKNOWLEDGEMENT}

The research of the first author has been fully supported by H.E.C. Pakistan under NRPU project 7906. 


\section{REFERENCES}

[1] M. Ali and A. R. Khan, "Generalized integral Mercer's inequality and integral means," Journal of Inequalities and Special Functions, vol. 10, no. 1, pp. 60-76, 2019.

[2] E. Anjidani, "Jensen-Mercer Operator Inequalities Involving Superquadratic Functions," Mediterr. J. Math., vol. 15, p. article 31, 2018, doi: 10.1007/s00009-017-1058-81660-5446/18/020001-12.

[3] E. Anjidani and M. R. Changalvaiy, "Reverse Jensen-Mercer type operator inequalities," Electron. J. Linear Algebra, vol. 31, pp. 87-99, 2016, doi: 10.13001/1081-3810.3058.

[4] S. Benbernou, S. Gala, and M. Ragusa, "On the regularity criteria for the 3D magnetohydrodynamic equations via two components in terms of BMO space," Mathematical Methods in the Applied Sciences, vol. 37, pp. 2320-2325, 2014, doi: 10.1002/mma.2981.

[5] H. Budak, T. Tunc, and M. Z. Sarikaya, "On Hermite-Hadamard type inequalities for f-convex function,” Miskolc Math. Notes, vol. 20, pp. 169-191, 2019, doi: 10.18514/MMN.2019.2436.

[6] S. S. Dragomir and R. P. Agarwal, "Two inequalities for differentiable mappings and applications to special means of real numbers and to trapezoidal formula," Applied Mathematics Letters, vol. 11, no. 5, pp. 91-95, 1998, doi: 10.1016/S0893-9659(98)00086-X.

[7] S. S. Dragomir and C. E. M. Pearce, "Selected Topics on Hermite-Hadamard Inequalities and Applications," RGMIA Monographs, Victoria University, 2000.

[8] G. Farid, "Existence of an integral operator and its consequences in fractional and conformable integrals," Open Journal of Mathematical Sciences, vol. 3, pp. 210-216, 2019, doi: 10.30538/oms2019.0064.

[9] S. Gala and M. A. Ragusa, "Logarithmically improved regularity criterion for the Boussinesq equations in Besov spaces with negative indices," Applicable Analysis, vol. 95, pp. 1271-1279, 2016, doi: 10.1080/00036811.2015.1061122.

[10] D. Gao, B. Xi, Y. Wu, and B. Guo, "On integral inequalities of HermiteHadamard type for coordinated r-mean convex functions," Miskolc Math. Notes, vol. 20, pp. 873-885, 2019, doi: 10.18514/MMN.2019.2828.

[11] A. Gözpinar, "Some Hermite-Hadamard type inequalities for convex functions via new fractional conformable integrals and related inequalities," AIP Conference Proceedings, vol. 1991, no. 1, p. 020006, 2018, doi: 10.1063/1.5047879.

[12] F. Jarad, E. Ugurlu, T. Abdeljawad, and D. Baleanu, "On a new class of fractional operators," Advances in Difference Equations, vol. 2017, pp. 121-128, 2017, doi: 10.1186/s13662-017-1306$\mathrm{z}$.

[13] M. Kian and M. S. Moslehian, "Refinements of the operator Jensen-Mercer inequality," Electron. J. Linear Algebra, vol. 26, pp. 742-753, 2013, doi: https://doi.org/10.13001/1081-3810.1684.

[14] A. A. Kilbas, "Hadamard-type fractional calculus," Journal of the Korean Mathematical Society, vol. 38, no. 6, pp. 1191-1204, 2001.

[15] A. A. Kilbas, H. M. Srivastava, and J. J. Trujillo, Theory and Applications of Fractional Differential Equations. Elsevier Science \& Technology, 2006. [Online]. Available: https://www.ebook.de/de/product/22933924/a_a_kilbas_theory_and_applications_of_ fractional_differential_equations.html

[16] A. Matkovic, J. Pečarić, and I. Perić, “A variant of Jensen's inequality of Mercer's type for operators with applications," Linear Algebra Appl., vol. 418, pp. 551-564, 2006, doi: 10.1016/j.laa.2006.02.030.

[17] A. M. Mercer, “A Variant of Jensens Inequality," J. Ineq. Pure and Appl. Math, vol. 4, no. 4, p. Article 73, 2003.

[18] D. S. Mitrinović, J. E. Pečarić, and A. M. Fink, Classical and new inequalities in analysis. Dordrecht: Kluwer Academic Publishers, 1993. 
[19] H. R. Moradi and S. Furuichi, "Improvement and Generalization of Some Jensen-Mercer-Type Inequalities," Journal of Math. Ineq, vol. 14, p. 377-383, 2020, doi: 10.7153/jmi-2020-14-24.

[20] M. Niezgoda, "A generalization of Mercers result on convex functions," Nonlinear Analysis, vol. 71, p. 277, 2009, doi: 10.1016/j.na.2009.01.120.

[21] S. Özcan and İ. İşcan, "Some new Hermite-Hadamard type inequalities for s-convex functions and their applications," Journal of Inequalities and Applications, vol. 2019, no. 1, p. 201, 2019, doi: 10.1186/s13660-019-2151-2.

[22] M. A. Ragusa and A. Tachikawa, "Regularity for minimizers for functionals of double phase with variable exponents," Adv. Nonlinear Anal., vol. 9, pp. 710-728, 2020, doi: 10.1515/anona-20200022 .

[23] M. Z. Sarikaya and N. Alp, "On Hermite-Hadamard-Fejer type integral inequalities for generalized convex functions via local fractional integrals," Open Journal of Mathematical Sciences, vol. 3, pp. 273-284, 2019, doi: 10.30538/oms2019.0070.

[24] M. Z. Sarikaya, E. Set, H. Yaldiz, and N. Basak, "Hermite-Hadamard's inequalities for fractional integrals and related fractional inequalities," Mathematical and Computer Modelling, vol. 57, pp. 2403-2407, 2013.

[25] M. Z. Sarikaya and H. Yildirim, "On Hermite-Hadamard type inequalities for RiemannLiouville fractional integrals," Miskolc Math. Notes, vol. 17, pp. 1049-1059, 2016, doi: 10.1016/j.mcm.2011.12.048.

Authors' addresses

Saad Ihsan Butt

COMSATS University Islamabad, Lahore Campus, Pakistan

E-mail address: saadihsanbuttegmail.com

\section{Ahmet Ocak Akdemir}

Ağrı İbrahim Çeçen University, Department of Mathematics, Faculty of Science and Letters, Ağrı, Turkey

E-mail address: aocakakdemiregmail.com

Jamshed Nasir

COMSATS University Islamabad, Lahore Campus, Pakistan

E-mail address: jnasir143@gmail.com

\section{Fahd Jarad}

Department of Mathematics, Cankaya University, Etimesgut 06790, Ankara, Turkey

E-mail address: fahdecankaya.edu.tr 